\title{
Analysis of transonic buffet using dynamic mode decomposition
}

\author{
Antje Feldhusen-Hoffmann ${ }^{1} \cdot$ Christian Lagemann $^{1} \cdot$ Simon Loosen $^{1} \cdot$ Pascal Meysonnat $^{1} \cdot$ Michael Klaas $^{1}$. \\ Wolfgang Schröder ${ }^{1}$
}

Received: 30 March 2020 / Revised: 3 November 2020 / Accepted: 2 December 2020 / Published online: 15 March 2021

(c) The Author(s) 2021

\begin{abstract}
The buffet flow field around supercritical airfoils is dominated by self-sustained shock wave oscillations on the suction side of the wing. Theories assume that this unsteadiness is driven by a feedback loop of disturbances in the flow field downstream of the shock wave whose upstream propagating part is generated by acoustic waves. High-speed particle-image velocimetry measurements are performed to investigate this feedback loop in transonic buffet flow over a supercritical DRA 2303 airfoil. The freestream Mach number is $M_{\infty}=0.73$, the angle of attack is $\alpha=3.5^{\circ}$, and the chord-based Reynolds number is $\operatorname{Re}_{c}=1.9 \times 10^{6}$. The obtained velocity fields are processed by sparsity-promoting dynamic mode decomposition to identify the dominant dynamic features contributing strongest to the buffet flow field. Two pronounced dynamic modes are found which confirm the presence of two main features of the proposed feedback loop. One mode is related to the shock wave oscillation frequency and its shape includes the movement of the shock wave and the coupled pulsation of the recirculation region downstream of the shock wave. The other pronounced mode represents the disturbances which form the downstream propagating part of the proposed feedback loop. The frequency of this mode corresponds to the frequency of the acoustic waves which are generated by these downstream traveling disturbances and which form the upstream propagating part of the proposed feedback loop. In this study, the post-processing, i.e., the DMD, is highlighted to substantiate the existence of this vortex mode. It is this vortex mode that via the Lamb vector excites the shock oscillations. The measurement data based DMD results confirm numerical findings, i.e., the dominant buffet and vortex modes are in good agreement with the feedback loop suggested by Lee.
\end{abstract}

Antje Feldhusen-Hoffmann

a.feldhusen@aia.rwth-aachen.de

1 Institute of Aerodynamics and Chair of Fluid Mechanics, RWTH Aachen University, Wüllnerstr. 5a, 52062 Aachen, Germany 


\section{Graphic abstract}

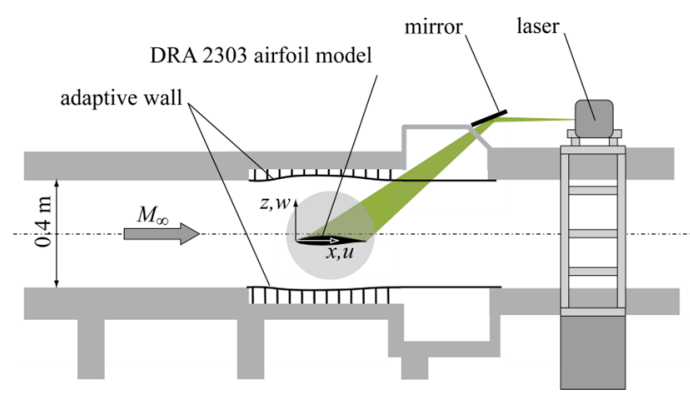

Fig. 1

Schematic of the trisonic wind tunnel and the PIV setup

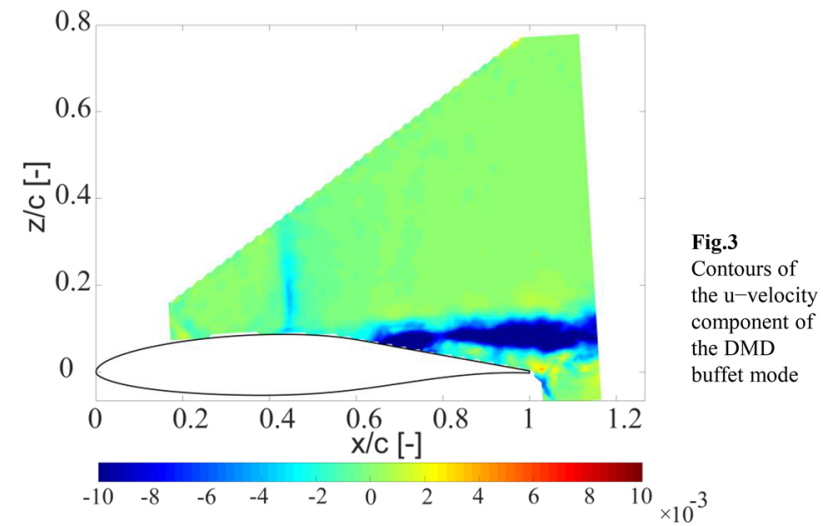

\section{Introduction}

A typical jet powered aircraft flies at transonic speed such that a supersonic region on the suction side of the airfoil develops which is terminated by a shock wave. For increasing Mach numbers or angles of attack, the pressure rise across the shock wave might become high enough to lead to a shock-induced separation of the boundary layer downstream of the shock wave. Within this transonic flight regime, highly unsteady flow including self-sustained periodic shock-wave oscillations, i.e., transonic buffet, might occur. The shock wave movement is a low frequency/large amplitude phenomenon. The resulting unsteady pressure distribution leads to unsteady loads acting on the wing structure. The aeroelastic response of the wing structure, the so-called buffeting, might lead to a critical state for the wing structure. To date, the mechanisms sustaining the shock-wave oscillations during buffet are not fully understood, yet. A detailed understanding of the buffet mechanisms are necessary to develop precise prediction methods for the onset of buffet, to shift the buffet

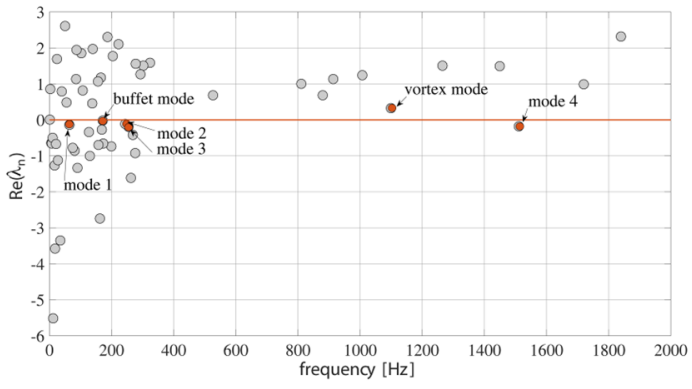

Fig. 2

Spectrum of the SP-DMD analysis for $\gamma=533.7$ in the physical frequency domain. Growth/decay rate of DMD modes, eigenvalues on the red line are stable.

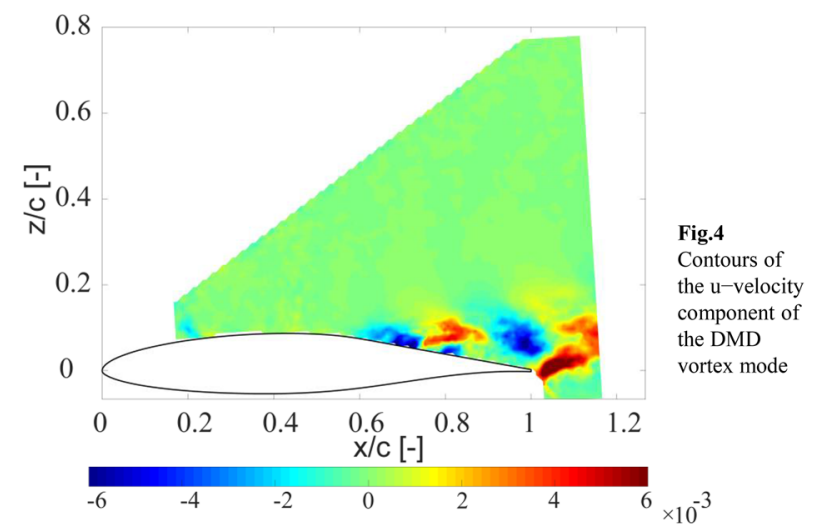

boundary to higher Mach numbers or angles of attack, and to find strategies to damp or even suppress buffet, which will enhance the operational performance of an aircraft. A comprehensive review on self-sustained shock wave oscillations on airfoils at transonic speeds has been given by Giannelis et al. (2017).

Despite intensive research, the mechanisms leading to buffet are still discussed controversially. Furthermore, the existing theories and models are usually restricted to special airfoils and flow characteristics.

For supercritical airfoils, a widely recognized description of the self-sustaining shock wave oscillation has been given by Lee (1990). His theory gives an explanation for self-sustained shock wave oscillations and a method to estimate the shock-wave-oscillation frequency for buffet flows around supercritical airfoils where the shock wave oscillates sinusoidally on the upper airfoil surface and induces a complete boundary-layer separation downstream. The basic idea is that disturbances which propagate up- and downstream within the flow field downstream of the shock wave form a feedback loop. According to Lee, the oscillating shock wave generates large-scale turbulent 
structures that propagate downstream and generate pressure waves while passing over the sharp trailing edge of the airfoil. These pressure waves travel also upstream and exchange energy with the shock wave, enhancing its oscillatory motion.

Xiao et al. (2006), Deck (2005), and Hartmann et al. (2012, 2013a, b), found excellent agreement of their numerical and experimental results with Lee's theory.

Hartmann et al. (2013a) refined the feedback model proposed by Lee (1990). The experiments by Hartmann et al. (2013a) revealed that the sound waves generated at the trailing edge and presenting the upstream propagating part of the feedback loop possess a high frequency which is about ten times higher than the shock-wave-oscillation frequency. Therefore, there has to be another low-frequency mechanism present in the flow field which forces the shock wave to oscillate at a frequency much lower than the trailing-edge noise. It is expected by Hartmann et al. (2013a) that the sound pressure level (SPL) of the sound waves originating at the trailing edge varies with a frequency that corresponds to the buffet frequency, i.e., the shock oscillation frequency. On the one hand, the relative velocity between the incoming flow and the oscillating shock wave is higher when the shock wave moves upstream. It triggers stronger disturbances which convect downstream towards the trailing edge. Hence, acoustic waves of an elevated SPL are generated which force the shock wave to move upstream while interacting with it. On the other hand, the shock wave is weaker when it moves downstream and excites weaker disturbances which convect downstream towards the trailing edge. As a consequence, acoustic waves with a lower SPL are generated which allow the shock wave to move back to its downstream position while interacting with it. In other words, the SPL of the trailing-edge noise and the frequency of the shock movement are supposed to be coupled. The findings of Feldhusen-Hoffmann et al. (2018) obtained from wind-tunnel experiments confirmed the expected variation of the SPL in the trailingedge region of the airfoil during buffet. Crouch et al. (2009) performed URANS simulations of the transonic flow around a symmetrical NACA 0012 profile for varying freestream Mach numbers and angles of attack, and a chord-based Reynolds number of $\operatorname{Re}_{c}=10^{7}$. A global stability analysis of the transonic airfoil flow was carried out to predict the onset of flow instabilities. A set of linearized equations was deduced from the Navier-Stokes equations which forms an eigenvalue problem governing the complex frequency and the shape of the global modes. The origin of buffet was predicted by the onset of an instability. The stability boundary as a function of the Mach number and angle of attack is in very good agreement with the boundary obtained from experimental data by McDevitt and Okuno (1985) for Mach numbers below 0.8 . The shape of the fluctuating streamwise velocity component of the unstable mode shows a coupled movement of the shock wave and the boundary layer downstream of the shock wave. On the one hand, the shape of the pressure fluctuations of the unstable mode reveals pressure perturbations originating near the shock wave foot moving upward along the shock wave and finally forward into the sonic zone. On the other hand, pressure fluctuations move downstream behind the shock wave, intensify, spread around the trailing edge, and propagate along the airfoil's pressure side until they enter into the sonic zone upstream of the shock wave. The observed pressure wave propagation is qualitatively different from the buffet model proposed by Lee (1990).

In this study, it is attempted to give further insight into the mechanisms of the buffet phenomenon. The buffet flow field around a supercritical airfoil is analyzed by dynamic mode decomposition (DMD). The velocity data are determined in wind tunnel experiments.

The dynamic mode decomposition (Schmid 2010) is a data-based technique which decomposes the underlying sequence of flow data into spatio-temporal coherent structures, i.e., dynamic modes. Each dynamic mode is associated with a single characteristic frequency, a growth or decay rate and an amplitude. On the one hand, DMD can be used for a reduced-order representation of the flow field, since the full dynamic system can be projected onto a subspace spanned by several extracted modes. On the other hand, it is an effective tool to search for physical mechanisms describing the underlying flow field evolution. There are many decomposition methods, the most common of which are the proper orthogonal decomposition (POD) method (Lumley 1967) and the global stability analysis. The POD modes generally are multi-frequential and the modes are sorted by their energy content, which in general does not allow any conclusion about the dynamical importance. The dynamic modes, however, are orthogonal in time and contain therefore only a single temporal frequency. Since the dominant features of the buffet phenomenon are related to characteristic frequencies, the decomposition by DMD is more suitable than the decomposition by POD. Furthermore, the decay rate of the dynamic modes reveals transient modes which are less dynamically important. The global stability analysis relies on the knowledge of the governing equations of the dynamical system under investigation. It is therefore not appropriate for the decomposition of experimental data. DMD, however, relies on the evaluation of time-discrete snapshot flow data and is therefore suitable to analyze experimental data. The dynamic modes can be regarded as a generalization of the global stability modes for nonlinear flow dynamics (Schmid 2010). If the underlying flow is linear or linearized, the dynamic modes are equivalent to the global modes resulting from the global stability analysis (Schmid 2010). This discussion shows the DMD technique to be an appropriate means for analyzing nonlinear dynamics of flow fields 
which are available as velocity snapshots. The assignment of dynamic modes to a single frequency will allow the revelation of possible couplings of flow structures. Since the dynamic modes are associated with a growth or decay rate, transient quickly decaying modes, which are of less dynamical importance, can be identified. The formalisms of DMD will be given in Sect. 3 .

Since its introduction, DMD has extensively been used for the investigation of flow fields comprising jets, shockwave/turbulent boundary layer interaction, wakes of airfoils and high-speed trains, wind turbine flow, and thermo-acoustic instabilities. These and other examples can be found in the comprehensive list of Rowley and Dawson (2017) compiled in their review on model reduction for flow analysis and control.

Several researchers applied DMD to the transonic buffet airfoil flow (Masini et al. 2018; Ohmichi et al. 2018; Kou and Zhang 2017; Kou et al. 2018; Gao et al. 2017; Poplingher et al. 2019). In these studies, the input data are pressure snapshots obtained numerically by unsteady Reynolds-averaged Navier-Stokes equations simulations (URANS) using the Spalart-Allmaras turbulence model. Masini et al. (2018) and Ohmichi et al. (2018) analyzed the three-dimensional shock buffet flow of swept wings. Only a few studies applied DMD to the two-dimensional shock buffet flow (Kou and Zhang 2017; Kou et al. 2018; Gao et al. 2017; Poplingher et al. 2019). The investigations comprise flows around a NACA 0012 airfoil (Kou and Zhang 2017; Kou et al. 2018; Gao et al. 2017) and around a supercritical RA16SC1 airfoil (Poplingher et al. 2019).

Kou and Zhang (2017) and Kou et al. (2018) performed flow reconstruction and prediction of periodic dynamics from the initial unstable transient flow solution based on dynamic modes. Besides the time-invariant mode, they found dominant modes related to the shock wave movement and its coupling to the boundary layer. However, the buffet mechanism was not further examined.

Gao et al. (2017) realized an active control to suppress buffet in an unstable steady airfoil flow being perturbed by trailing edge flap oscillations. DMD was used as a complementary tool to detect dominant frequencies and coherent structures. They found the dominant periodic dynamic modes to result from the shock wave movement and the respective frequency to be the shock-wave-oscillation frequency or multiples of it. Since the dominant buffet mode is damped but present under active flow control, the authors stated that buffet occurs due to a global instability, supporting the findings of Crouch et al. (2009).

Poplingher et al. (2019) used DMD of the transonic supercritical RA16SC1 airfoil flow to find dominant dynamic modes describing the buffet flow, to reconstruct the flow field, and to investigate the response of the pre-buffet unstable flow to excitations with the vertical velocity component.
The frequencies of the dominant periodic dynamic modes are multiples of the shock-wave-oscillation frequency and the mode shapes present pressure gradients in the shock travel region and in the boundary layer downstream of the shock wave. The authors stated, however, that trailing edge vortex shedding cannot be identified with certainty due to restrictions given by the URANS modeling and the grid resolution in the wake area. The time-space modal history of the buffet mode revealed similar pressure propagation as found by Crouch et al. (2009). The investigation of the modal excitation showed buffet-like mode shapes oscillating with a frequency close to that during buffet onset. The dominant buffet mode is damped but this damping decreases to zero at buffet onset conditions.

The present study applies DMD to experimental velocity data of the transonic buffet flow around the supercritical DRA 2303 airfoil. The analysis evidences individual dominant periodic dynamics of the buffet flow, comprising not only the shock wave oscillation but also the sharp trailing edge vortex shedding. The dominant modes are extracted by combining a priori knowledge of dominant frequencies resulting from preceding investigations with results from sparsity-promoting DMD (SP-DMD). Since the transonic buffet flow exhibits some periodic features, DMD will help to capture the dominant frequency information and stability characteristics. In this context, DMD is not used to reconstruct or predict the flow behavior but to gain further insight into the mechanisms of buffet.

In contrast to the previously mentioned studies, in this work measurement data determine the input snapshots for the DMD analysis of a two-dimensional transonic buffet flow field. The results will show that experimentally obtained velocity distributions are suitable as input data for the DMD analysis of this particular flow case. An a priori investigation is performed to show the sensitivity of the SP-DMD results with regard to the number of snapshots as input data. Since the velocity data are recorded by a high sampling rate, it is possible unlike in the study of Poplingher et al. (2019) to detect the trailing-edge vortex shedding by DMD. Other than in the studies (Masini et al. 2018; Ohmichi et al. 2018; Kou and Zhang 2017; Kou et al. 2018; Gao et al. 2017; Poplingher et al. 2019), in which only indications of the theory of Crouch et al. (2009) were found, the current measurement data based DMD results corroborate Lee's theory (Lee 1990).

The paper is structured as follows. The Introduction is followed by the description of the experimental setup in Sect. 2 . In Sect. 3, the DMD approach is concisely presented. In the results Sect. 4, some characteristics of the DRA 2303 buffet flow field, which are known from previous investigations, are described in Sect. 4.1 to allow a comparison of the former findings with the results determined by the DMD analysis. The DMD results of the DRA 2303 airfoil buffet flow are 


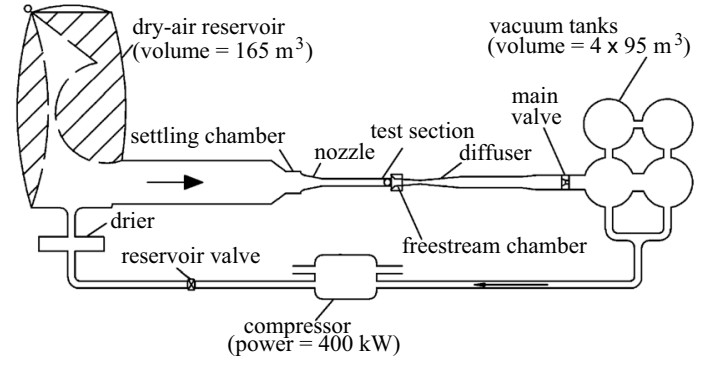

Fig. 1 Sketch of the trisonic wind tunnel

given in Sect. 4.2, followed by concluding remarks given in Sect. 5.

\section{Experimental setup}

\subsection{Wind tunnel}

All measurements are performed in the trisonic vacuum storage wind tunnel of the Institute of Aerodynamics, RWTH Aachen University. A sketch of the tunnel is shown in Fig. 1. A compressor evacuates four vacuum tanks with an overall volume of $380 \mathrm{~m}^{3}$ downstream of the closed test section. The air from the tanks is guided through a silica gel based drier and stored in a settling reservoir upstream of the test section under ambient conditions. The drier ensures that the relative humidity of the air is kept below $4 \%$ to minimize any influence of the humidity on the shock wave position (Binion 1988). To initiate a run, the main quick-acting valve downstream of the diffuser opens and the air flows through the test section. The turbulence intensity of the flow entering the test section is less than $1 \%$. Since the tunnel works intermittently, the measurement time with stable flow conditions is limited to two to three seconds depending on the Mach number. The Mach number can be varied from $M_{\infty}=0.3$ to $M_{\infty}=4.0$, whereas the Reynolds number depends on the Mach number and on the ambient conditions in the dry-air reservoir. Therefore, the unit Reynolds number $\mathrm{Re} / L$ is restricted to the range of $12 \times 10^{6} \mathrm{~m}^{-1} \leq \mathrm{Re} / L \leq 14 \times 10^{6} \mathrm{~m}^{-1}$ for the transonic Mach number regime.

During the run of the tunnel, the instantaneous Mach number is recorded by unsteady pressure data using a pressure sensor mounted upstream of the test section. When the desired Mach number is reached a trigger signal is released with a short delay such that the PIV acquisition starts. This ensures that the wind tunnel flow is stable during the measurement time.

The test section possesses a square cross section of $0.4 \mathrm{~m} \times 0.4 \mathrm{~m}$ and a length of $1.41 \mathrm{~m}$. For the investigation of transonic flows, the flexible upper and lower adaptive walls of the test section simulate unconfined flow conditions by solving the 1D-Cauchy integral based on the steady pressure distribution along each wall measured during the previous run.

The inflow parameters of the wind tunnel were set to the freestream Mach number $M_{\infty}=0.73$ and the angle of attack $\alpha=3.5^{\circ}$. The chord-based Reynolds number, which depends on the Mach number $M_{\infty}$ and on the ambient conditions, was $\operatorname{Re}_{c}=1.9 \times 10^{6}$. Under these conditions the DRA 2303 airfoil model exhibits distinct shock wave oscillations, i.e., buffet (Hartmann et al. 2013a).

\subsection{Airfoil model}

The flow over a supercritical laminar type DRA 2303 profile with a chord length of $0.15 \mathrm{~m}$ is measured. It is a twodimensional model spanning the complete test section width. The relative ratio of the airfoil thickness to chord length is $14 \%$ which leads to a blockage of about $5 \%$ when mounted inside the adaptive test section. Note that for the wing model with constant cross-section in the spanwise direction the buffet characteristics differ from those for three-dimensional wings, where the most severe large-scale unsteadiness occures at the wing tip and the flow frequencies are approximately one order of magnitude higher and more broadband (Roos 1985).

The airfoil model is made of two carbon fiber laminate sandwich shells and incorporates a steel beam inside which ensures a rigid mounting in the test section. Laminar to turbulent transition is imposed by a $117 \mu \mathrm{m}$ thick zigzag stripe located at $5 \%$ chord on both suction and pressure side. The width of the zigzag transition stripe is $5 \mathrm{~mm}$. A photo of the airfoil model is shown in Fig. 2.

\subsection{Particle-image velocimetry}

The PIV setup installed at the trisonic wind tunnel is shown in Fig. 3. Prior to each test run, a Laskin nozzle generator seeds the dry air inside the tunnel reservoir with polydisperse Di-Ethyl-Hexyl-Sebacate (DEHS) droplets with a mean diameter of less than $1 \mu \mathrm{m}$. Inside the test section, the particles are illuminated by a Nd:YLF Darwin Duo 527100-M high-speed laser. Since two oscillators are installed

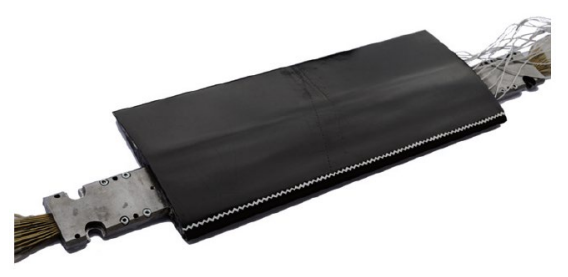

Fig. 2 Photograph of the supercritical DRA 2303 airfoil 

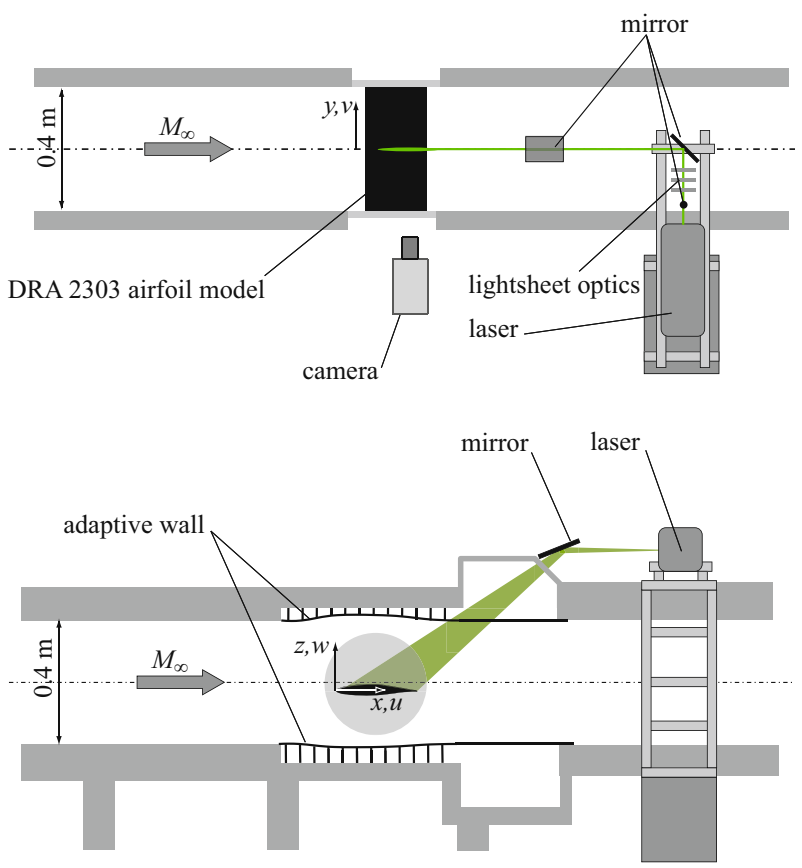

Fig. 3 Schematic of the trisonic wind tunnel and the PIV setup [top view (top), side view (bottom)] with the airfoil model installed

inside a single laser head, very high repetition rates can be achieved. The pulse width is less than $210 \mathrm{~ns}$, which is adequate for the underlying flow. The total pulse energy at a repetition rate of $1 \mathrm{kHz}$ is $60 \mathrm{~mJ}$. The laser is installed outside and separated from the tunnel to protect it from tunnel vibrations. A system of mirrors and a light-sheet optic span the laser beam and deflect it to the test section. The beam can enter the test section through a slot in the freestream chamber. The light of the laser spans a $1 \mathrm{~mm}$ thick vertical streamwise measurement plane in the midspan region of the airfoil's suction side.

A Photron Fastcam SA5 high-speed camera with $1024 \times 1024$ pixel resolution is used to record the forward scattered light of the seeding particles. The camera is installed perpendicular to the measurement plane. The images are acquired using the frame straddling technique and the pulsation of the lasers and the opening of the camera shutters are synchronized with an ILA GmbH synchronizer. The sampling frequency is $f_{\mathrm{s} \text {.PIV }}=4000 \mathrm{~Hz}$.

The correlation of the raw images was performed using PIVView from ILA GmbH by applying a multi-grid interrogation method. The correlation was preceded by a background-subtraction for image pre-processing. The correlation was followed by a vector outlier detection using a maximum displacement filter and a dynamic mean test filter. Table 1 lists the hardware, the settings, and the resulting data of the PIV setup.

The uncertainty in determining the flow velocity is dominated by the uncertainty in the estimation of the particle displacement. This is due to the findings that for the given laser pulse separation time of $9 \mu \mathrm{s}>1 \mu \mathrm{s}$ the error from the laser pulse separation time can be neglected (Lazar et al. 2010), and for reliable calibrations the magnification and calibration uncertainties can also be neglected (dos Santos et al. 2019). Given that the particle image diameter equals 3 pixels or more and applying multi-pass interrogation algorithms employing image deformation techniques, the typical error value for the displacement is on the order of 0.1 pixel (Adrian et al. 2011; Adrian 1986; Raffel et al. 2018; Westerweel 1993, 1997, 2000). Thus, a conservative estimation of the measurement error yields uncertainties of $0.7 \%$ in terms of the incoming flow velocity.

\section{Dynamic mode decomposition}

In the DMD flow field data, e.g., velocity data $\boldsymbol{v}(\boldsymbol{x}, t)$ are decomposed into $n$ spatial modes $\boldsymbol{\phi}_{n}(\boldsymbol{x})$ with the amplitudes $a_{n}$, and the complex frequencies $\lambda_{n}$

$\boldsymbol{v}(\boldsymbol{x}, t)=\sum_{n} a_{n} \mathrm{e}^{\lambda_{n} t} \boldsymbol{\phi}_{n}(\boldsymbol{x})$.

The flow field data are collocated from numerical simulations or measurements as a sequence of $N$ snapshots, e.g., velocity fields, equispaced in time with the time step $\Delta t$ in the time interval $t=[0,(N-1) \Delta t]$. The snapshot sequence is column-wise stored in a data matrix
Table 1 Specifics of the particle-image velocimetry setup

\begin{tabular}{llll}
\hline Hardware and settings & & Resulting data \\
\hline Camera & Fastcam SA5 & Measurement area & $0.15 \leq x / c \leq 1.16$ \\
Lens & Nikkor $85 \mathrm{~mm} \mathrm{f} / 1.8 \mathrm{D}$ & Resolution & $1024 \times 888$ pixel \\
Laser & Darwin Duo 527-100-M & Initial window size & $96 \times 96$ pixel \\
Light-sheet thickness & $1 \mathrm{~mm}$ & Final window size & $24 \times 24$ pixel \\
Laser pulse separation time & $9 \mu \mathrm{s}$ & Window overlap & $50 \%$ \\
$f_{\mathrm{s}, \text { PIV }}$ & $4000 \mathrm{~Hz}$ & Final vector spacing & $1.78 \mathrm{~mm}$ \\
Number of captured buffet cycles & $\sim 265$ & & \\
\hline
\end{tabular}


$\boldsymbol{V}_{1}^{N}=\left[\boldsymbol{v}_{1}, \boldsymbol{v}_{2}, \boldsymbol{v}_{3}, \ldots, \boldsymbol{v}_{N}\right], \quad \boldsymbol{v}_{i} \in \mathbb{R}^{M}$,

where the subscript denotes the first entry and the superscript the last entry in the sequence. The spatial dimension $M$ of the data is typically much larger than the number of snapshots $N$, i.e., $M \gg N$. A linear mapping is assumed which is approximately the same over the full sampling interval

$\boldsymbol{v}_{i+1}=\boldsymbol{A} \boldsymbol{v}_{i}, \quad i \in\{1, \ldots, N-1\}$

allowing the sequence to be described as a Krylov sequence (Greenbaum 1997; Trefethen and Bau III 1997)

$V_{1}^{N}=\left[v_{1}, A v_{1}, A^{2} v_{1}, \ldots, A^{N-1} v_{1}\right]$

If the snapshots are derived from flow fields with nonlinear dynamics, this assumption is equal to a linear tangent approximation. The sought characteristics of the dynamical process described by the sequence $\boldsymbol{V}_{1}^{N}$ can be extracted from the matrix $\boldsymbol{A}$ by calculating its eigenvectors and eigenvalues. However, $\boldsymbol{A}$ is usually hard to determine due to its vast size of $M \times M$ with $M \gg N$. Using DMD, a low-order representation of $\boldsymbol{A}$ is determined which equally captures the dynamics of the snapshot sequence.

Schmid (2010) introduced a robust implementation by extracting the dynamic characteristics from a 'full and robust' DMD matrix $\boldsymbol{F}_{\text {dmd }} \in \mathbb{C}^{N-1 \times N-1}$ being a low-dimensional representation of the linear intersnapshot operator $\boldsymbol{A}$ on the subspace spanned by the basis $\boldsymbol{U}$

$\boldsymbol{F}_{\mathrm{dmd}} \equiv \boldsymbol{U}^{*} \boldsymbol{A} \boldsymbol{U}$

with $(\cdot)^{*}$ denoting the conjugate transpose of a matrix. Robustness is achieved by determining $\boldsymbol{U}$ via a singular value decomposition (SVD) of the snapshot sequence

$\boldsymbol{V}_{1}^{N-1}=\boldsymbol{U} \boldsymbol{\Sigma} \boldsymbol{W}^{*}$.

The quantities, $\boldsymbol{U} \in \mathbb{C}^{M \times(N-1)}$ and $\boldsymbol{W} \in \mathbb{C}^{(N-1) \times(N-1)}$ are the left and right singular vectors and the matrix $\boldsymbol{\Sigma} \in \mathbb{C}^{(N-1) \times(N-1)}$ contains the singular values of the snapshot sequence $\boldsymbol{V}_{1}^{N-1}$.

The matrix $\boldsymbol{F}_{\mathrm{dmd}}$ describes the dynamics of the snapshot sequence, which has originally been described by the matrix $\boldsymbol{A}$ in Eq. (3), on the subspace spanned by the basis $\boldsymbol{U}$ such that

$\boldsymbol{x}_{i+1}=\boldsymbol{F}_{\mathrm{dmd}} \boldsymbol{x}_{i}$.

The eigenvalue decomposition of the DMD matrix

$\boldsymbol{F}_{\mathrm{dmd}} \boldsymbol{Y}=\mu \boldsymbol{Y}$

yields the so-called Ritz eigenvalues $\mu_{n}$ and the eigenvectors $\boldsymbol{y}_{n}$.

The dynamic modes are obtained by multiplying the right singular vectors of the snapshot sequence $\boldsymbol{V}_{1}^{N-1}$ with the $n$th eigenvector $\boldsymbol{\phi}_{n}:=\boldsymbol{U} \boldsymbol{y}_{n}$ and their corresponding amplitude based on the first snapshot is given by $a_{n}:=z_{n}^{*} x_{1}$ with $\left\{z_{1}^{*}, \ldots, z_{N-1}^{*}\right\}$ being the eigenvectors of $\boldsymbol{F}_{\mathrm{dmd}}^{*}$ and $\boldsymbol{x}_{1}$ representing the initial condition. The snapshots are approximated by a linear combination of the dynamic modes

$\boldsymbol{v}_{i}=\sum_{n=1}^{N-1} a_{n}\left(\mu_{n}\right)^{i-1} \boldsymbol{\phi}_{n}, \quad i \in\{1, \ldots, N-1\}$,

which can be written in matrix form for the discrete snapshot sequence

$$
\begin{aligned}
& \underbrace{\left[\boldsymbol{v}_{1}, \boldsymbol{v}_{2}, \ldots, \boldsymbol{v}_{N-1}\right]}_{V_{1}^{N-1}}=\underbrace{\left[\boldsymbol{\phi}_{1}, \boldsymbol{\phi}_{2}, \ldots, \boldsymbol{\phi}_{N-1}\right]}_{\boldsymbol{\Phi}} \\
& \underbrace{\left(\begin{array}{ccccc}
a_{1} & & & \\
& a_{2} & & \\
& & \ddots & \\
& & & a_{N-1}
\end{array}\right)}_{\boldsymbol{D}_{a}=\operatorname{diag}\{a\}} \underbrace{\left(\begin{array}{cccc}
1 & \mu_{1} & \cdots & \mu_{1}^{N-1} \\
1 & \mu_{2} & & \mu_{2}^{N-1} \\
\vdots & \vdots & & \vdots \\
1 & \mu_{N-1} & & \mu_{N-1}^{N-1}
\end{array}\right)}_{\boldsymbol{V}_{\text {and }}} .
\end{aligned}
$$

The matrix filled with the geometric progression of the Ritz eigenvalues is called Vandermonde matrix $\boldsymbol{V}_{\text {and }}$ and describes the temporal evolution of the modes. The matrix $\boldsymbol{D}_{a}$ contains the optimized amplitudes based on all snapshots $a_{n}$ of the modes which can be found by minimizing the objective function

$\underset{a}{\operatorname{minimize}}\left\|\boldsymbol{V}_{1}^{N-1}-\boldsymbol{\Phi} \boldsymbol{D}_{a} \boldsymbol{V}_{\text {and }}\right\|_{F}^{2}$

with $\|\cdot\|_{F}$ being the Frobenius norm. For input data from experiments or numerical simulations contaminated by noise and/or other uncertainties, the matrix $\boldsymbol{\Sigma}$ usually has a full rank such that the DMD analysis yields the maximum number of $(N-1)$ modes and eigenvalues. The classical optimized DMD generally extracts $r \leq(N-1)$ modes with $r$ being the rank of the matrix $\boldsymbol{\Sigma}$, which results from the SVD of the snapshot sequence given in Eq. (6). Knowing the complex quantities $\left\{a_{n}, \mu_{n}, \boldsymbol{\phi}_{n}\right\}$, it follows from Eqs. (1) and (9) that

$\mu_{n}=r_{n} \mathrm{e}^{\mathrm{i} \Theta_{n}}=\mathrm{e}^{\lambda_{n} \Delta t}$ such that

$\lambda_{n}=\frac{\ln \left(\mu_{n}\right)}{\Delta t}=\underbrace{\frac{\mathfrak{R}\left(\ln \left(\mu_{n}\right)\right)}{\Delta t}}_{\text {growth/decay }}+\mathrm{i} \underbrace{\frac{\Im}{\Delta t}\left(\ln \left(\mu_{n}\right)\right)}_{\text {angular frequency }}=\lambda_{n, \mathrm{r}}+\mathrm{i} \lambda_{n, \mathrm{i}}$.

Thus, the real part $\lambda_{\mathrm{r}}$ of the complex frequency contains information about the growth or decay rate of the DMD mode. The dynamic mode is stable for decaying $\lambda_{\mathrm{r}}$, i.e., $\lambda_{\mathrm{r}}<0$, and unstable for an excited $\lambda_{\mathrm{r}}$, i.e., for $\lambda_{\mathrm{r}}>0$. For $\lambda_{\mathrm{r}}=0$, the mode is a stable mode which is present throughout the whole snapshot sequence. Transient modes, which do not contribute significantly to the data sequence, are 
characterized by a high decay rate $\lambda_{\mathrm{r}} \ll 0$ and therefore by complex Ritz eigenvalues $\mu_{n}$ with an absolute value of $r_{n} \ll 1$ [see Eq. (12)]. Periodic stable Ritz eigenvalues are characterized by an absolute value of $r_{n}=1$. The imaginary part $\lambda_{\mathrm{i}}$ of the complex frequency contains information about the temporal periodicity of the DMD mode, given in the unit [rad]. The frequency in the unit $[\mathrm{Hz}]$ is $f=\mathfrak{J}\left(\ln \left(\mu_{n}\right)\right) /(2 \pi \Delta t)$. For $\lambda_{\mathrm{i}}=0$, the respective frequency is zero meaning that the dynamic mode represents the mean mode. Due to real input data, the Ritz eigenvalues $\mu_{n}$ with a non-zero frequency appear as pairs of complex conjugates of the same frequency and decay or growth rate. Detailed information on the DMD method and its application can be found in Schmid (2010) and in recent review papers (Rowley and Dawson 2017; Taira et al. 2017, 2020).

Identifying the dynamically relevant modes is usually not straightforward and makes the 'manual' mode selection very tedious in practice. The standard approach to select the dominant dynamic modes by their amplitude $a_{n}$ is often not reasonable since transient modes are likely to appear with artificially high amplitudes as will be shown in the following section. There exist, however, some pre- and post-processing approaches to identify dynamically relevant modes, which can be applied depending on the problem under investigation and the main objective of the analysis. In this study, DMD mode selection based on the comparison to the spectral analyses and on the sparsity-promoting DMD (SP-DMD) have been applied. They will be explained in the following.

A preceding spectral analysis by, e.g., FFT or the PSD function of the data, can help to find distinct frequencies, and thus eigenvalues, which are dominant in the flow field and should be further analyzed by DMD.

The SP-DMD, introduced by Jovanović et al. (2014), is an extension of the standard DMD algorithm which seeks a subset of DMD modes that have the most substantial influence on the quality of the approximation of the snapshot sequence. Note, however, that the SP-DMD also might select transient modes. The user defines a tradeoff between the approximation error and the number of extracted modes. A constraint consisting of a penalty term for the number of non-zero elements in the amplitude vector $\boldsymbol{a}$ is introduced to the least-squares problem given in Equation (11) and forces a sparse solution

$\underset{a}{\operatorname{minimize}}\left\|\boldsymbol{V}_{1}^{N-1}-\boldsymbol{\Phi} \boldsymbol{D}_{a} \boldsymbol{V}_{\text {and }}\right\|_{F}^{2}+\gamma \sum_{n=1}^{N-1}\left|a_{n}\right|$

The parameter $\gamma$ is the positive regularization parameter. The larger its value, the more zero elements exist in the amplitude vector $\boldsymbol{a}$. This sparsity structure is fixed in the following and the optimal amplitudes are computed for the resulting subset of dynamic modes. Jovanović et al. (2014) developed an efficient algorithm to solve the regularized least-squares problem given in Eq. (13) which uses the alternating direction method of multipliers (ADMM). The ADMM algorithm requests the user to choose a maximum number of iterations, to set the augmented Lagrangian parameter, or step-size parameter, $\rho$, which also determines the rate of convergence of the algorithm, and to give the absolute and relative tolerances $\epsilon_{\mathrm{abs}}$ and $\epsilon_{\text {rel }}$ for the minimization steps. The step-size parameter $\rho>0$ influences the convergence speed of the algorithm (Ghadimi et al. 2012). Furthermore, large values of $\rho$ diminish the primal residual and augment the dual residual of the optimization problem and the opposite is true for small values of $\rho$. It has been shown, however, that the ADMM method converges for all values of $\rho$ (Boyd et al. 2011). The primal and dual residuals of the optimization problem can be used to define stopping criteria $\epsilon_{\text {abs }}$ and $\epsilon_{\text {rel }}$ for the ADMM algorithm (Boyd et al. 2011). The details are given in Jovanović et al. (2014). Tu et al. (2014) addressed the appropriate selection of dominant dynamic modes and noted that the SP-DMD approach proposed by Jovanović et al. (2014) is well suited for the DMD analysis.

\section{Results}

In this section, the results of the experimental study of the DRA 2303 buffet flow field are presented. First, a general description of the flow field is given in Sect. 4.1, i.e., the main flow features and dominant frequencies are emphasized. This overview is used to justify the selection of relevant dynamic modes based on the comparison to spectral analyses as mentioned in Sect. 3. Furthermore, this overview allows to evaluate whether the dominant features of the buffet flow and the proposed feedback loop (Lee 1990) are correctly detected by the SP-DMD analysis.

\subsection{Description of the DRA 2303 buffet flow field}

The main characteristics of the feedback loop proposed by Lee (1990) present in the buffet flow field around the supercritical DRA 2303 airfoil have been previously investigated experimentally by Hartmann et al. (2013a) and Feldhusen et al. $(2013,2015)$ for the same freestream conditions of $M=0.73$ and $\alpha=3.5^{\circ}$. This study is a direct continuation of the aforementioned investigations. Note that exactly the same data set as in Feldhusen et al. (2013) is used. In the following, just a concise description of the primary features is given. Note that the chord length of the DRA 2303 airfoil model used by Hartmann et al. (2013a) was $200 \mathrm{~mm}$ and thereby larger than the chord length of the DRA 2303 model used in Feldhusen et al. $(2013,2015)$, and in this study. However, since the reduced frequency $\omega^{\star}=2 \pi f c / u_{\infty}$ is the same, the results 
can be compared. A shock wave occurs on the suction side of the DRA 2303 airfoil that induces a boundary layer separation which extends to the sharp trailing edge. The shock wave oscillation frequency $f_{\text {buffet }}=170 \mathrm{~Hz}$ is illustrated in Fig. 4.

The broadband shock wave motion is coupled to the pulsation of the recirculation region with a small phase lag, i.e., the shock wave movement precedes the pulsation of the boundary layer. When the shock wave moves upstream, the size of the recirculation region grows normal to the airfoil upper surface such that the extension of the recirculation region is largest when the shock wave is located most upstream. When the shock wave moves downstream, the extension of the recirculation region diminishes and is smallest when the shock wave is located most downstream. The shock wave motion provokes downstream propagating disturbances which generate upstream traveling disturbances when passing over the trailing edge. Following the analysis in Feldhusen et al. (2013), the wave propagation characteristics of these upstream propagating disturbances in the flow field on the suction side of the

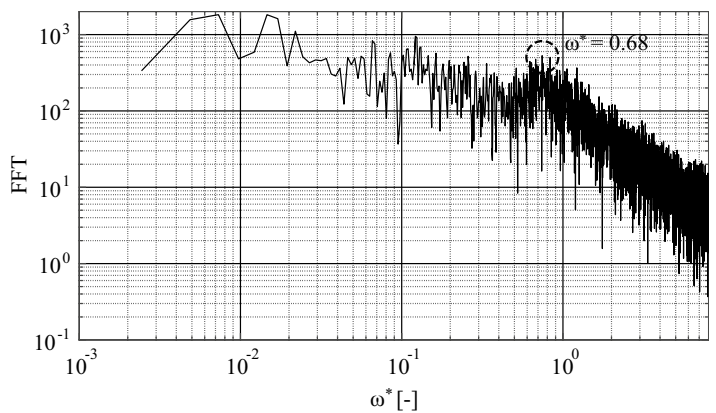

Fig. 4 Amplitude spectrum of the FFT analysis of the signal of a pressure transducer located in the shock wave region at $x / c=0.42$ on the upper side of the DRA 2303 airfoil from Ref. Feldhusen et al. (2013). The frequency is given as reduced frequency $\omega^{*}=2 \pi f c / u_{\infty}$ airfoil are known from the spatio-temporal correlation of unsteady flow velocity data

$R_{n m}(x, \tau)=\frac{\overline{U_{n}^{\prime}(t) \cdot U_{m}^{\prime}(t+\tau)}}{\sqrt{\overline{U_{n}^{\prime 2}} \cdot \overline{U_{m}^{\prime 2}}}}$

illustrated in Fig. 5a. The fluctuation quantities of the absolute velocities $U_{n}^{\prime}(t)$ and $U_{m}^{\prime}(t+\tau)$ separated by the time delay $\tau$ and located at the positions $x_{n} / c=$ const. and varying $x_{m} / c$ along the time-averaged streamline shown in Fig. $5 \mathrm{~b}$ are correlated. The propagation speed of the disturbances is determined by the slope of the line connecting the high correlation values in the spatio-temporal correlation plots and the frequency of the disturbances can be deduced from the distance of the periodic pattern of the lines connecting high correlation values. This analysis yields disturbances propagating upstream with a frequency $f_{\mathrm{TE}}=1100$ $\mathrm{Hz}$ and a velocity $85 \mathrm{~m} \mathrm{~s}^{-1}$. These disturbances form the upstream propagating part of the suggested feedback loop. When reaching the shock wave, they maintain its oscillation. Since the frequency of the upstream propagating trailingedge noise differs from the frequency of the shock wave oscillation, it is assumed (Hartmann et al. 2013a) that the trailing-edge noise varies in its strength with a frequency corresponding to the shock wave oscillation frequency. The stronger sound pressure level forces the shock wave to move upstream while a lower sound pressure level allows the shock wave to move downstream. The moving shock wave causes downstream propagating vortices of different strengths depending on the relative velocity of the shock wave with respect to the incoming flow. When these vortices pass over the trailing edge, they provoke noise with a varying sound pressure level. This closes the proposed feedback loop mechanism. Further details of the feedback loop mechanisms are discussed, e.g., in Hartmann et al. (2013a) and Feldhusen-Hoffmann et al. (2018).
Fig. 5 Correlation of the absolute velocities $R_{n m}(x, \tau)$ along a time-averaged streamline (Feldhusen et al. 2013)

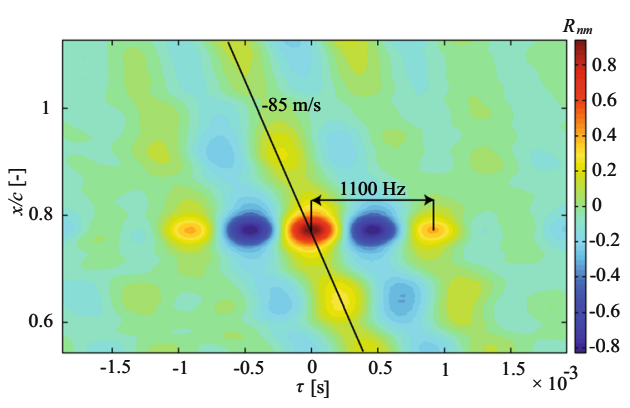

(a) Correlation of the absolute velocities along the time-averaged streamline; $x_{n} / c=0.79$, data band-pass filtered between 700 and $1400 \mathrm{~Hz}, 5 \times$ temporal interpolated.

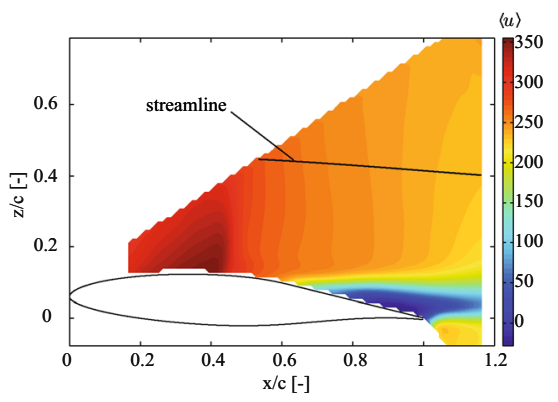

(b) Time-averaged velocity field, the instantaneous absolute velocity distributions along the streamline are used for the correlation. 


\subsection{DMD analysis of the DRA 2303 buffet flow}

In this section, the dynamic mode decomposition of the experimentally determined DRA 2303 transonic buffet flow field is presented. The $u$-velocity field and the $v$-velocity field are arranged on top of each other in a single snapshot matrix. Since the range of the velocity data varies widely, the data are scaled in a preprocessing step by normalizing them with the maximum value of the $u$-and $v$-velocity component. First, the number of relevant modes is reduced by SP-DMD, presented in Sect. 4.2.1. Two dynamically important modes which represent main features of the proposed feedback loop (Lee 1990), namely the buffet mode and the vortex mode, are pointed out in Sects. 4.2.2 and 4.2.3. Finally, further stable modes are presented in Sect. 4.2.4.

\subsubsection{Reducing the number of modes by SP-DMD}

The appropriate number of snapshots that should be fed into the DMD algorithm is determined by balancing the stability of the modes and the number of dynamic modes. Transient phenomena due to the onset and offset of buffet during the start and the shutdown of the flow in the wind tunnel are not taken into account in the snapshots for the DMD such that the flow field of the snapshots is periodic. Therefore, no physically transient modes that are related to the buffet phenomenon are expected to be present in the snapshot sequence. On the one hand, there exists a lower limit for the number snapshots that should be fed into the DMD algorithm to correctly resolve the low-frequency shock oscillation motion and on the other hand, the interpretation of the DMD results and the extraction of dominant modes becomes more complex with an increasing number of snapshots.

As stated in the previous section, transient Ritz eigenvalues are characterized by an absolute value of $r_{n} \ll 1$, i.e., they are inside the unit disk when being plotted in the complex plane. Stable Ritz eigenvalues defined by $r_{n}=1$ accumulate on the unit circle. Figure 6a shows the Ritz eigenvalues plotted in the complex plane together with the unit circle for $N_{z}=1900$ snapshots. Several values for $N_{z}$ have been tested. For $N_{z}=1600$, the saturation point is reached such that for 1600 snapshots or more there is no significant decrease of unstable Ritz eigenvalues. To be on the safe side, $N_{z}=1900$ snapshots have been chosen, which corresponds to about 80 buffet cycles. These snapshots are not taken from the beginning or end but from the intermediate measurement time. They are used for the following analysis.

The spectrum of eigenvalues is symmetric with respect to the real axis. The eigenvalue $(\mathfrak{R}(\mu), \mathfrak{J}(\mu))=(1,0)$ is associated with a frequency of zero and represents the time-invariant mode, i.e., the mean mode. Since most of the Ritz eigenvalues cluster on the unit disk, only a few unstable dynamic modes are present in the spectrum. In Fig. 6b, the amplitudes of the modes are plotted versus the growth/decay rates. The red line marks a growth/decay rate of zero, i.e., stable modes. The modes with high amplitudes are associated with high decay rates. Since these modes vanish quickly at the beginning of the snapshot sequence due to their very high decay rate, they are transient. Strongly decaying modes with high amplitudes are often present in DMD analyses, see, e.g., (Jovanović et al. 2014, Fig. 4c) . That is, even when analyzing a stable or periodic flow where no transient flow dynamics are present, this artefact of high amplitude quickly decaying modes appears. Therefore, these modes are not related to the periodic buffet phenomenon, which is present during the whole snapshot sequence. In other words, ranking the relevance of the dynamic modes by their amplitude is not reasonable.

Instead, dominant dynamic modes have been extracted by SP-DMD (Jovanović et al. 2014). Overall, 100 values in the interval $\left[1,10^{5}\right]$ for the regularization parameter $\gamma$ for nonzero amplitudes have been tested. The optimal choice of the step-size parameter $\rho$ is still subject of ongoing research (see, e.g. Ghadimi et al. 2012). Here, $\rho=1$ is used as proposed by Jovanović et al. (2014). Since the convergence rate was sufficient, an optimization by varying this parameter was not necessary. Boyd et al. (2011) stated $\epsilon_{\text {rel }}=10^{-3}$ or $10^{-4}$ as reasonable values for the relative tolerance. In this study $\epsilon_{\text {rel }}=10^{-4}$ has been chosen. The maximum absolute truncation error was set to $10^{-6}$.

Figure 7a shows the degradation of performance with increasing values of $\gamma$. The performance loss is given by

$\Pi_{\text {loss }}=100 \frac{\left\|\boldsymbol{V}_{1}^{N}-\boldsymbol{\phi} \boldsymbol{D}_{a} \boldsymbol{V}_{\text {and }}\right\|_{F}}{\left\|\boldsymbol{V}_{1}^{N}\right\|_{F}} \%$.

The performance loss is thus the residual $\left\|\boldsymbol{V}_{1}^{N}-\boldsymbol{\phi} \boldsymbol{D}_{a} \boldsymbol{V}_{\text {and }}\right\|_{F}$ of the representation of the snapshots $\boldsymbol{V}_{1}^{N}$ resulting from SPDMD normalized by $\left\|\boldsymbol{V}_{1}^{N}\right\|_{F}$. Note that the output of the SP-DMD analysis is sensitive to the choice of the regularization parameter $\gamma$ for non-zero amplitudes, i.e., the number of non-zero amplitudes $N_{z}$. Therefore, the approach for finding a suitable value for the regularization parameter is driven by the results obtained from Hartmann et al. (2013a) and Feldhusen et al. (2013). For the simplest case $N_{z}=1$, the time-invariant mode representing the mean mode is selected by SP-DMD. Up to $N_{z}=19$, only low-frequency modes $(f<50 \mathrm{~Hz})$ are selected. The characteristic buffet frequency $f_{\text {buffet }}=170 \mathrm{~Hz}$ appears for $N_{z}>29$. When the number of non-zero amplitudes $N_{z}$ is further increased, the frequency spectrum of the selected modes expands to lower and higher frequencies. For the finally chosen value of $N_{z}=115$, the high frequency of the trailing-edge noise $f_{\mathrm{TE}}=1100 \mathrm{~Hz}$ appears for the first time in the DMD spectrum. Since the previously determined characteristic frequencies $f_{\text {buffet }}=170 \mathrm{~Hz}$ and $f_{\mathrm{TE}}=1100 \mathrm{~Hz}$ are present in the SP-DMD spectrum for $N_{z}=115$, these snapshots have 
Fig. 6 Spectrum of the optimized DMD analysis for $N_{z}=1900$

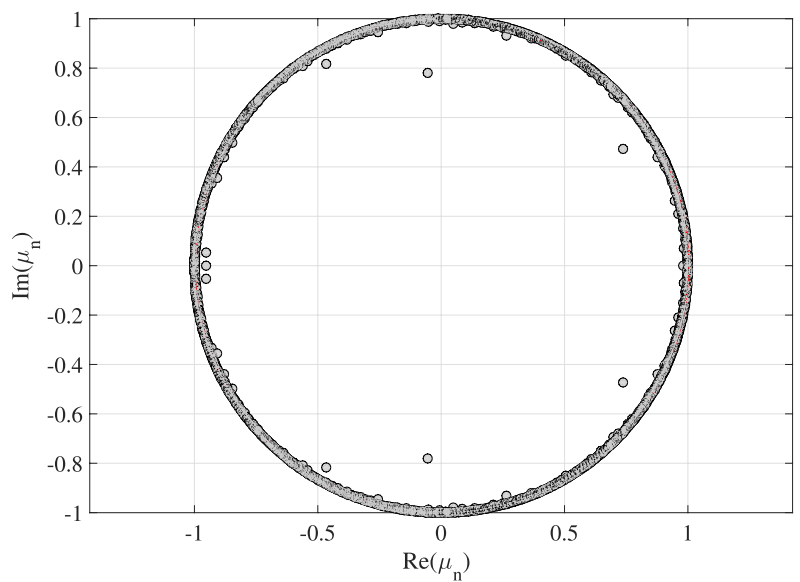

(a) Ritz eigenvalues plotted together with the unit circle (dashed red line); eigenvalues on the unit circle are stable throughout the snapshot sequence.

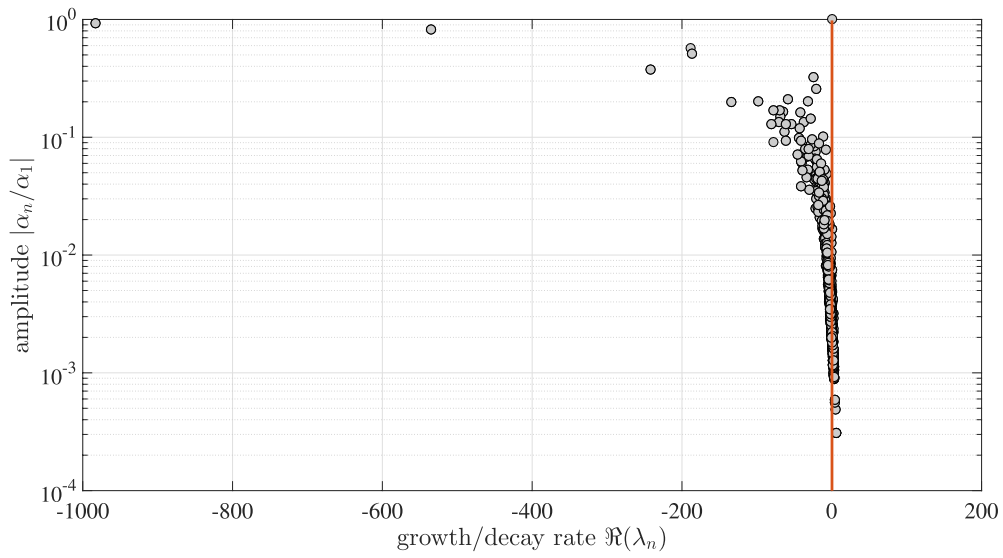

(b) Dependence of the absolute value of the normalized DMD amplitudes on the growth/decay rate of the dynamic modes; eigenvalues lying on the red line are stable throughout the snapshot sequence. been chosen for the following investigations. This results in a regularization parameter of $\gamma=533.7$, that also defines the limit below which the number of modes increases rapidly (see Fig. 7b), and a performance loss below $10 \%$, i.e., $\Pi_{\text {loss }}=9.3211 \%$ (see Fig. 7a).

The spectrum of the SP-DMD analysis for $\gamma=533.7$ in the physical frequency domain is given in the plots of Fig. 8. Since the eigenvalues appear as complex conjugate pairs with the same frequency, a one-sided representation with respect to the frequency is sufficient. The amplitudes $a_{n}$ in Fig. 8a are normalized by the amplitude of the timeinvariant mode $a_{1}$. The red horizontal line in Fig. 8b marks a growth/decay rate of zero such that eigenvalues lying on this line are stable throughout the snapshot sequence. Since the absolute values of the decay rates are overall much lower than in the spectrum for the large set of DMD modes with $N_{z}=1900$ given in Fig. 6b, it is evident that the SP-DMD with $N_{z}=115$ excludes the most transient modes from the spectrum.

\subsubsection{The buffet mode}

In the following, the dominant characteristic modes of the transonic DRA 2303 buffet flow are identified and analyzed. The dynamics of the buffet flow is identified by selecting the DMD modes whose growth or decay rates are close to zero.

In the range of the characteristic buffet frequency $f_{\text {buffet }}=170 \mathrm{~Hz}$, there is one marginally stable mode in Fig. 8b. This mode will be referred to as the buffet mode. Its decay rate is nearly zero and its respective frequency is $f=171.56 \mathrm{~Hz}$. The reconstruction of the buffet mode for the first time step is given in Fig. 9. The contours show the scaled dynamic mode, i.e., the spatial distribution $\boldsymbol{\Phi}(\boldsymbol{x})$ of the mode scaled by the respective amplitude $a$. The 


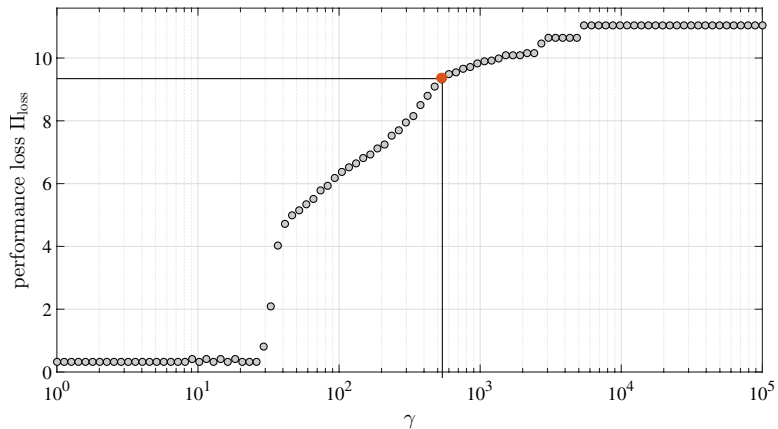

(a) Performance loss of the optimal vector of amplitudes.

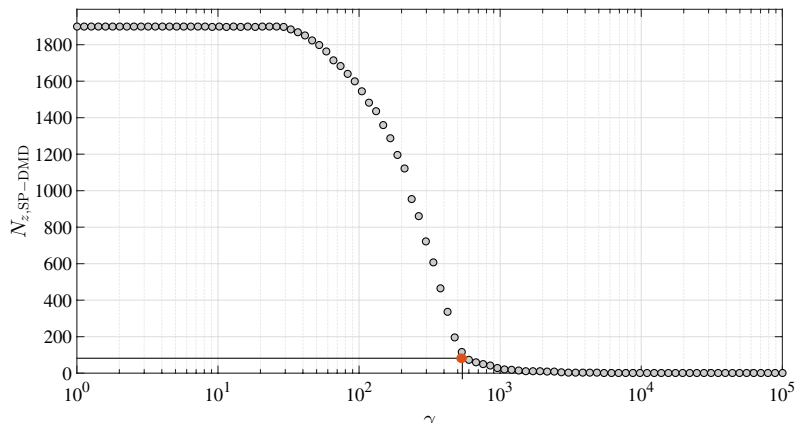

(b) Number of non-zero amplitudes.

Fig. 7 Performance of the SP-DMD algorithm as a function of the regularization parameter $\gamma$, the value for $\gamma$ defined in Fig. 7a is indicated by the red dots

$u$-velocity component of the mode (Fig. 9a) reveals that not only the shock motion is included in the buffet mode but also the pulsating recirculation region. Whereas the $u$-velocity component of the buffet mode is dominated by the shock wave motion and the pulsation of the recirculation region, the $w$-velocity component of the mode (Fig. 9b) has sign changes in the streamwise direction, which indicates a wavelike movement of the recirculation region.

The coupling between the shock wave motion and the pulsation of the recirculation region is further analyzed by examining the time-space modal history of the $u$-velocity component of the buffet mode in Fig. 10 for five characteristic stages of one oscillation cycle. Note that only the temporal reconstruction of the buffet mode is shown without superposing the mean mode. Therefore, the contours in the shock wave region do not indicate the speed of the shock wave motion, but the flow velocity in this area. At the first stage (Fig. 10a), there is a negative superimposed velocity in the shock region, meaning that the shock is located most upstream. The negative superimposed flow velocity in the recirculation region indicates a pronounced separation. When the shock moves downstream (Fig. 10b), the flow in the recirculation region accelerates first at the shock foot. At the third stage (Fig. 10c), the shock wave is located most downstream since there is a superimposed positive velocity in the shock region. Higher velocities of the flow in the recirculation region show that the separation of the boundary layer is less pronounced. At a later time (Fig. 10d), the shock wave travels upstream. The shock speed reduces and the flow in the recirculation region decelerates first at the shock foot. The shock motion and location cause the change of the extension of the recirculation region. Finally, the shock wave reaches again its most upstream position (Fig. 10e). The finding from Fig. 10, that variations related to the length scale of the recirculation region start at the shock foot, substantiates the result from Fig. 9 that the recirculation region grows and diminishes in a wave-like manner.

The $u$-velocity component of the buffet mode presents the main characteristics of the buffet flow directly visible in Schlieren images, see e.g. (Hartmann et al. 2012). Regarding the proposed feedback mechanism (Lee 1990), the pulsation of the recirculation region directly influences the sound pressure level interacting with the shock wave. Due to a larger separation region, the sound waves generated at the trailing edge undergo stronger refractions such that their sound pressure level weakens in comparison to a narrow separation region.

\subsubsection{The vortex mode}

In the SP-DMD mode spectra of Fig. 8, one pronounced mode can be found at the frequency of the sound waves originating at the sharp trailing edge $f_{\mathrm{TE}}=1100 \mathrm{~Hz}$ which will be referred to as the vortex mode since the trailingedge sound is produced by vortices passing over it. The growth rate of the vortex mode is near zero, indicating that this mode is marginally unstable, i.e., very slowly growing. As it is shown in the contour plots in Fig. 11, the reconstruction of the vortex mode consists of vortical structures primarily in the region of the flow recirculation. In the context of the proposed feedback mechanism (Lee 1990), these vortices present the downstream propagating part. They are produced by the moving shock wave, travel downstream, and pass over the trailing edge. Due to the interaction of this unsteady flow field with the trailingedge sound is emitted. For this vortex-driven sound, the perturbation of the vorticity-velocity cross product, the so-called Lamb vector, is the major sound source (Ewert and Schröder 2003). The strength of the moving shock wave depends on the relative velocity between the moving shock wave and the incoming flow (Gibb 1988), i.e., the shock strength increases while moving upstream. A stronger shock wave triggers stronger vortex roll-up such that the strength of the downstream propagating vortices varies. The measured $w$-velocity component of the buffet mode (see Fig. 9b) indicates that the vortices might be modulated in their strength by a frequency corresponding 
Fig. 8 Spectrum of the SPDMD analysis for $\gamma=533.7$ in the physical frequency domain; red dots highlight various modes

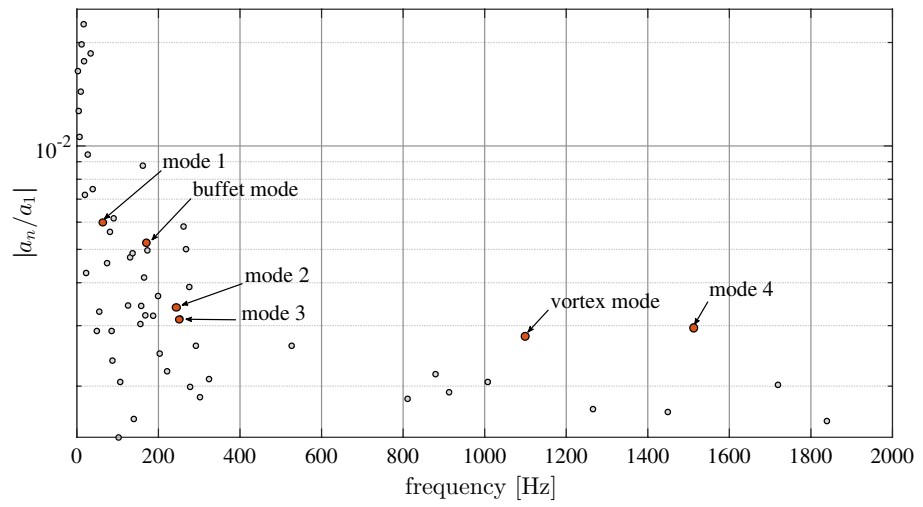

(a) Normalized DMD amplitude spectrum. For readability, the time-invariant mode is not plotted.

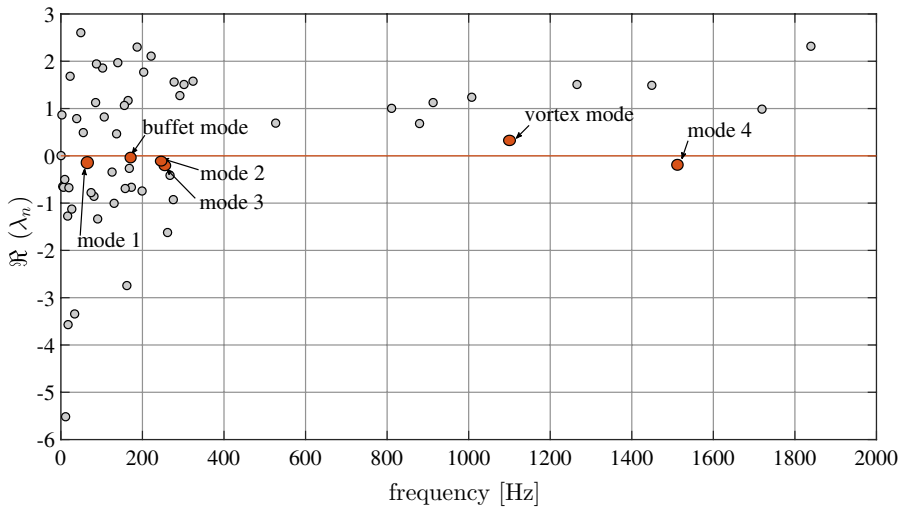

(b) Growth/decay rate of DMD modes, eigenvalues on the red line are stable.

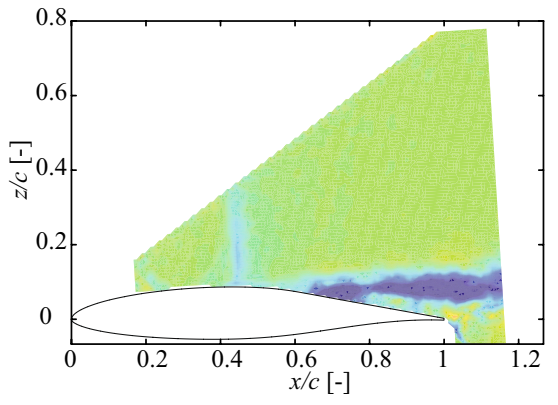

(a) $u$-velocity component.

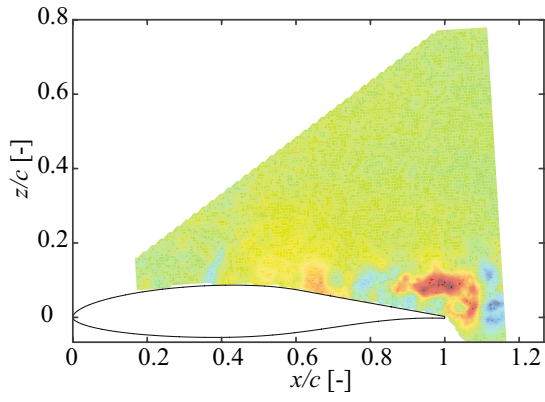

(b) $w$-velocity component.
Fig. 9 Contours of the $u$ - and $w$-velocity component of the DMD buffet mode with $f=171.56 \mathrm{~Hz}$ to the buffet frequency. Finally, vortices with changing strength will generate trailing-edge noise of a varying sound-pressure level which will maintain the shock wave movement. Note that the buffet mode and the vortex mode still exist for a perturbed snapshot data matrix.

\subsubsection{Further stable modes}

In Fig. 8b, further marginally stable periodic modes with a low decay rate which are more stable than the vortex mode indicated as mode 1 to mode 4 are present. The corresponding contours of the $u$ - and $w$-velocity component are plotted in Figs. 12, 13, 14 and 15. Mode 1 has a low frequency of $64 \mathrm{~Hz}$. It possesses the highest amplitude of all selected marginally stable periodic modes, followed by the buffet mode. The reconstruction of the $u$-velocity component of mode 1 includes the shock wave and the recirculation region, and the reconstruction of the $w$-velocity component of mode 1 reveals vortical structures such that overall, the reconstruction of mode 1 is very similar 
Fig. 10 Contours of the $u$-velocity component of temporal reconstruction with the DMD buffet mode in one buffet cycle; $\Delta t_{\text {buffet }}=1 / f_{\text {buffet }}=1 / 170 \mathrm{~Hz}$
Fig. 11 Contours of the $u$ and $w$-velocity component of the DMD vortex mode with $f=1100 \mathrm{~Hz}$

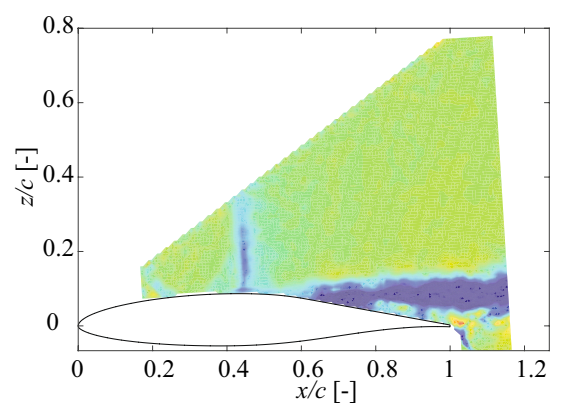

(a) Time instant $t$; shock wave in most upstream position $x / c=0.42$.

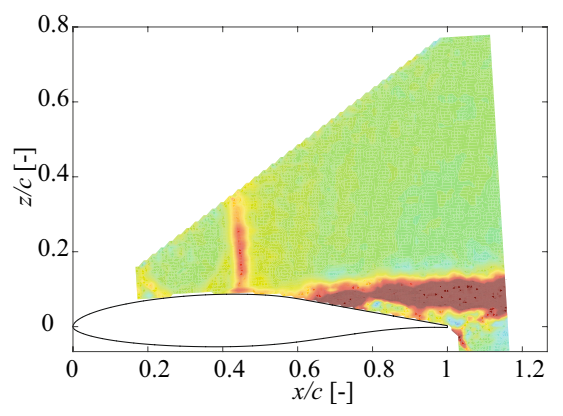

(c) Time instant $t+1 / 2 \Delta t_{\text {buffet }}$; shock wave in most downstream position $x / c=0.47$.

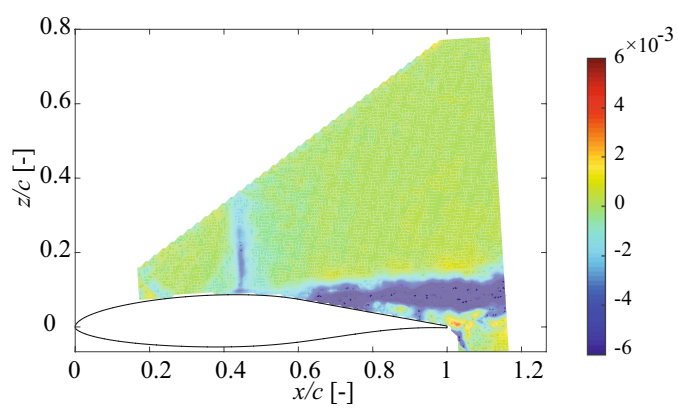

(e) Time instant $t+\Delta t_{\text {buffet }}$; shock wave in most upstream position $x / c=0.42$.

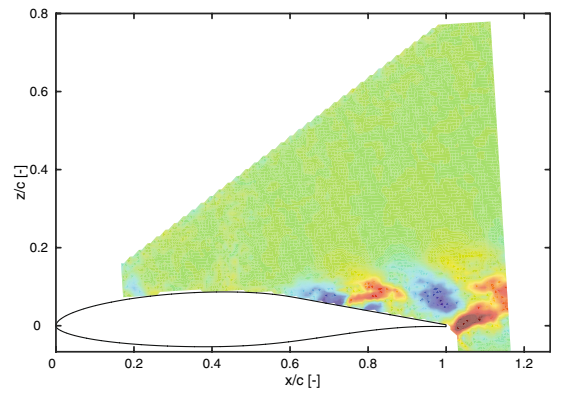

(a) $u$-velocity component.

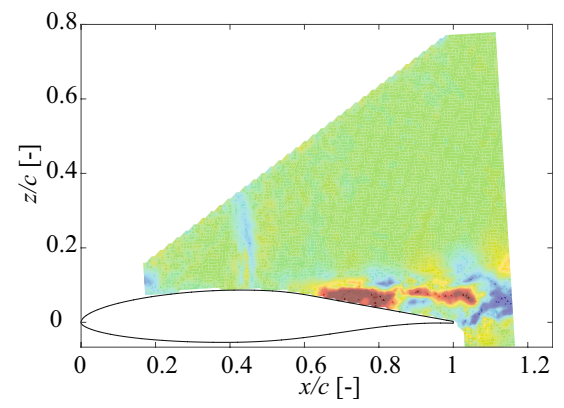

(b) Time instant $t+1 / 4 \Delta t_{\text {buffet }}$; shock wave moves downstream.

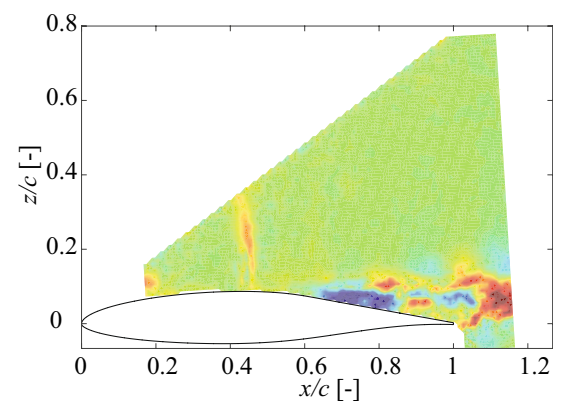

(d) Time instant $t+3 / 4 \Delta t_{\text {buffet }}$; shock wave moves upstream.

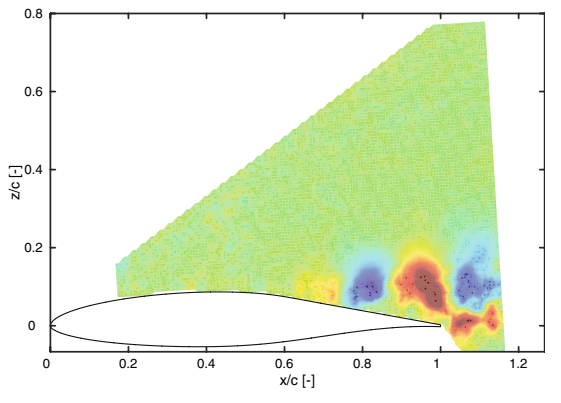

(b) $w$-velocity component.

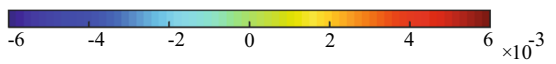

to the reconstruction of the buffet mode. Mode 2 and mode 3 illustrated in Figs. 13 and 14 are very similar to each other. Their frequency is $244 \mathrm{~Hz}$ and $253 \mathrm{~Hz}$. Their reconstructions include the shock wave and the recirculation region in the $u$-velocity component of mode 2 , and pronounced vortical structures. Mode 4 has a frequency of 
Fig. 12 Contours of the $u$ - and $w$-velocity component of DMD mode 1

Fig. 13 Contours of the $u$ - and $w$-velocity component of DMD mode 2

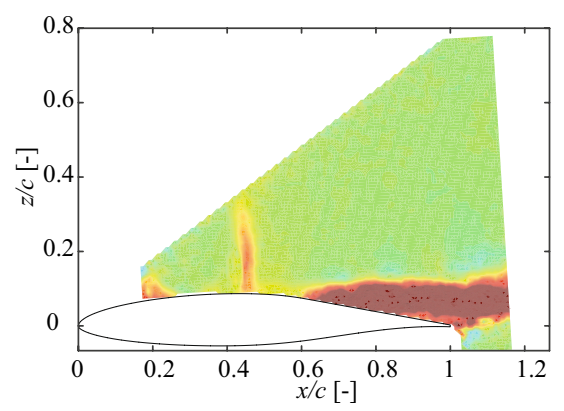

(a) $u$-velocity component.

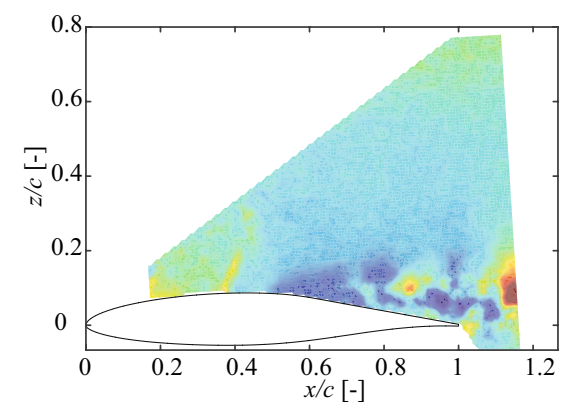

(b) $w$-velocity component.

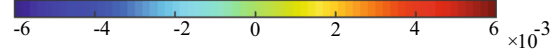

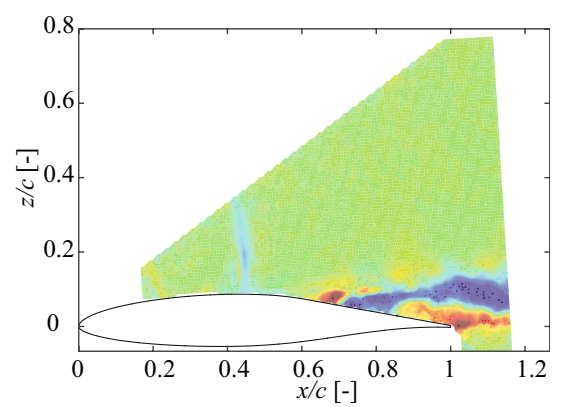

(a) $u$-velocity component.

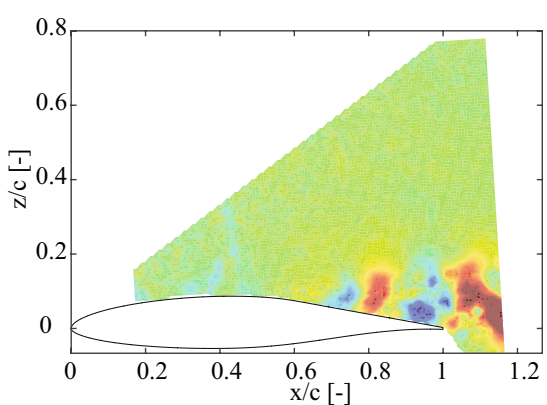

(b) $w$-velocity component.

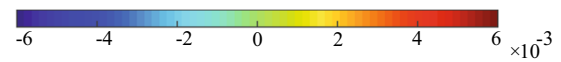

Fig. 14 Contours of the $u$ - and $w$-velocity component of DMD mode 3

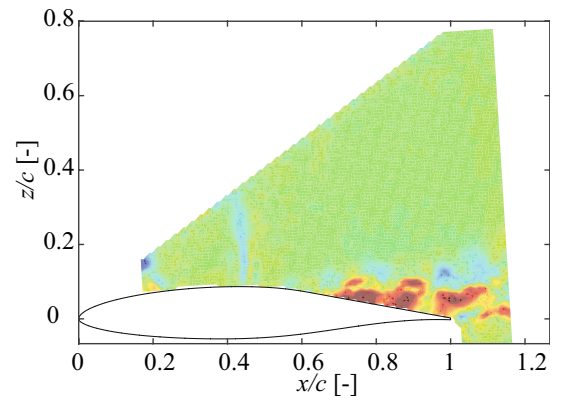

(a) $u$-velocity component.

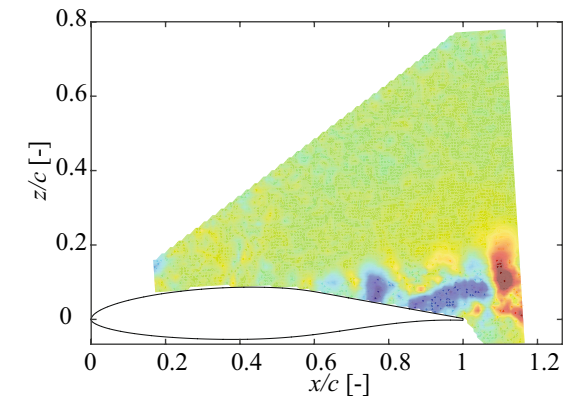

(b) $v$-velocity component.

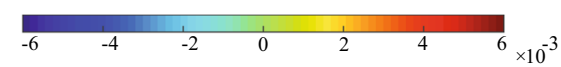

$1511 \mathrm{~Hz}$ and the corresponding spatial structures plotted in Fig. 15 are vortices in the separated boundary layer. Its amplitude is low compared to the other selected marginally stable modes, followed only by the vortex mode (see Fig.8a). Since the frequency and spatial distribution of mode 4 and the vortex mode are similar, it is supposed that they contribute to the same phenomenon of vortex roll-up inside the separated shear layer. Whereas further investigations are needed to clarify the role of mode 1 , it is supposed that modes 2 and 3 contribute to the overall buffet dynamics without characterizing any additional dynamic phenomena.

\section{Conclusion}

The transonic buffet flow field around a supercritical DRA 2303 airfoil model has experimentally been investigated by particle-image velocimetry measurements. For the first time, the experimentally obtained velocity data of a buffet flow 
Fig. 15 Contours of the $u$ - and $w$-velocity component of DMD mode 4

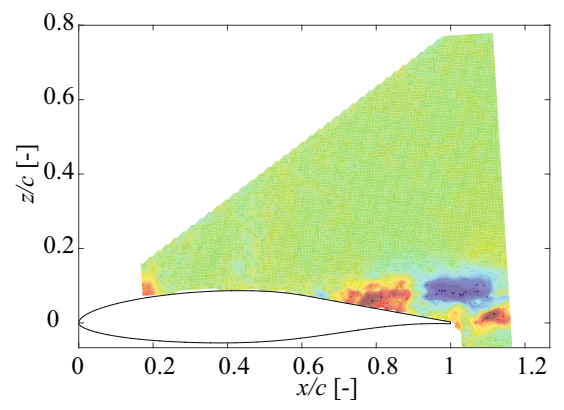

(a) $u$-velocity component.

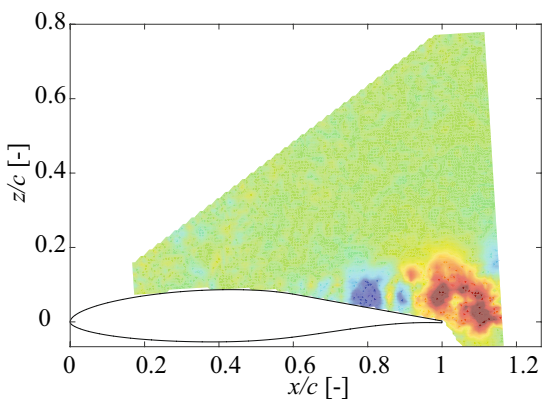

(b) $w$-velocity component

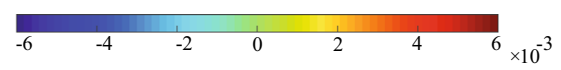

field are further processed to perform a sparsity-promoting dynamic mode decomposition (SP-DMD).

The physics of the buffet phenomenon around the DRA 2303 airfoil was thoroughly discussed, e.g., in Hartmann et al. (2013a) and in Feldhusen-Hoffmann et al. (2018), supporting the buffet model by Lee (1990). This model assumes that the shock wave oscillations are driven by an acoustic feedback loop consisting of a downstream propagating part represented by shock induced vortices and an upstream propagating part represented by sound waves which are generated when the downstream moving vortices pass over the trailing edge. The shock wave movement is supposed to be sustained by a varying SPL of the sound waves interacting with it.

From the previous studies, two dominant frequencies, the low frequency of the shock wave oscillation and the high frequency of the vortex shedding, are known. Using the present SP-DMD analysis these frequencies could be assigned to their spatial structures. The low-frequency buffet mode captures the coupling of the shock wave oscillation and the pulsation of the recirculation region. The downstream location of the shock wave is associated with a less pronounced separation of the boundary layer than the upstream location. Changes in the velocity of the flow in the recirculation region start at the shock foot and convect downstream towards the sharp trailing edge. This leads to a wave-like up- and down motion of the recirculation region, also visible in the $w$-velocity component of the buffet mode. Hartmann et al. (2013a) argued that the changing size of the recirculation region enhances the variation of the trailing-edge noise SPL. For a larger recirculation region, they found a lower wall-normal gradient of the streamwise velocity component indicating a lower vorticity which is the main driving mechanism of noise. Furthermore, the locally generated sound waves are weakened while propagating upstream since they undergo stronger interactions and refractions when the recirculation region is larger. This supports the idea of Lee (1990) and Hartmann et al. (2013a) that during buffet, the shock wave is forced to oscillate by the trailing-edge noise of varying SPL. Furthermore, the DMD substantiates the existence of the high-frequency vortex mode that excites the shock oscillations via the Lamb vector.

Four further marginally stable periodic modes have been identified. It is supposed that they are not related to additional singular physical features.

In brief, the study shows that experimentally obtained velocity distributions are suitable for the DMD analysis of the 2D buffet flow and can be used for the future analysis of $3 \mathrm{D}$ buffet flow. Since the velocity data are recorded by a high sampling rate, buffet generated trailing-edge vortex shedding is detected by measurement data based DMD, which was not discussed in the literature, yet. The experimental results confirm numerical findings, i.e., the dominant buffet and vortex modes are in good agreement with the feedback loop suggested by Lee (1990).

Acknowledgements This research was funded by the Deutsche Forschungsgemeinschaft within the research project "Numerical and Experimental Analysis of Shock Oscillations at the Shock-BoundaryLayer Interaction in Transonic Flow" (DFG SCHR 309/40-2).

Funding Open Access funding enabled and organized by Projekt DEAL.

Open Access This article is licensed under a Creative Commons Attribution 4.0 International License, which permits use, sharing, adaptation, distribution and reproduction in any medium or format, as long as you give appropriate credit to the original author(s) and the source, provide a link to the Creative Commons licence, and indicate if changes were made. The images or other third party material in this article are included in the article's Creative Commons licence, unless indicated otherwise in a credit line to the material. If material is not included in the article's Creative Commons licence and your intended use is not permitted by statutory regulation or exceeds the permitted use, you will need to obtain permission directly from the copyright holder. To view a copy of this licence, visit http://creativecommons.org/licenses/by/4.0/.

\section{References}

Adrian R (1986) Multi-point optical measurements of simultaneous vectors in unsteady flow-a review. Int J Heat Fluid Flow 7(2):127-145 
Adrian L, Adrian RJ, Westerweel J (2011) Particle image velocimetry. Cambridge University Press, Cambridge

Binion TW (1988) Potentials for pseudo-Reynolds number effects. In: Reynolds number effects in transonic flow. Technical report, AGARDograph 303, sec. 4

Boyd S, Parikh N, Chu E (2011) Distributed optimization and statistical learning via the alternating direction method of multipliers. Now Publishers Inc, Boston

Crouch JD, Garbaruk A, Magidov D, Travin A (2009) Origin of transonic buffet on aerofoils. J Fluid Mech 628:357-369

Deck S (2005) Numerical simulation of transonic buffet over a supercritical airfoil. AIAA J 43(7):1556-1566

dos Santos AAC, Childs M, Nguyen TD, Hassan Y (2019) Convergence study and uncertainty quantification of average and statistical PIV measurements in a matched refractive index $5 \times 5$ rod bundle with mixing vane spacer grid. Exp Therm Fluid Sci 102:215-231

Ewert R, Schröder W (2003) Acoustic perturbation equations based on flow decomposition via source filtering. J Comput Phys 188:365-398

Feldhusen A, Hartmann A, Klaas M, Schröder W (2013) Impact of alternating trailing-edge noise on buffet flows. In: 31st AIAA applied aerodynamics conference, San Diego, CA. pp 3028

Feldhusen A, Klaas M, Schröder W (2015) High-speed tomographic PIV measurements of buffet flow over a supercritical airfoil with artificially introduced sound waves. In: 11th international symposium on particle image velocimetry-PIV15 Santa Barbara, California

Feldhusen-Hoffmann A, Statnikov V, Klaas M, Schröder W (2018) Investigation of shock-acoustic-wave interaction in transonic flow. Exp Fluids 59(1):15

Gao C, Zhang W, Kou J, Liu Y, Ye Z (2017) Active control of transonic buffet flow. J Fluid Mech 824:312-351

Ghadimi E, Teixeira A, Shames I, Johansson M (2012) On the optimal step-size selection for the alternating direction method of multipliers. IFAC Proc Vol 45(26):139-144

Giannelis NF, Vio GA, Levinski O (2017) A review of recent developments in the understanding of transonic shock buffet. Prog Aerosp Sci 92:39-84

Gibb J (1988) The cause and cure of periodic flows at transonic speeds. In: Proceedings of the 16th congress of the international council of aeronautical sciences, Jerusalem, Israel, August-September 1988. pp 1522-1530

Greenbaum A (1997) Iterative methods for solving linear systems, vol 17. SIAM, Philadelphia

Hartmann A, Klaas M, Schröder W (2012) Time-resolved stereo PIV measurements of shock-boundary layer interaction on a supercritical airfoil. Exp Fluids 52:591-604

Hartmann A, Feldhusen A, Schröder W (2013a) On the interaction of shock waves and sound waves in transonic buffet flow. Phys Fluids 25(2):026101

Hartmann A, Klaas M, Schröder W (2013b) Coupled airfoil heave/pitch oscillations at buffet flow. AIAA J 51(7):1542-1552

Jovanović MR, Schmid PJ, Nichols JW (2014) Sparsitypromoting dynamic mode decomposition. Phys Fluids 26(2):0241031-02410322

Kou J, Zhang W (2017) An improved criterion to select dominant modes from dynamic mode decomposition. Eur J Mech B Fluid 62:109-129

Kou J, Le Clainche S, Zhang W (2018) A reduced-order model for compressible flows with buffeting condition using higher order dynamic mode decomposition with a mode selection criterion. Phys Fluids 30(1):016103

Lazar E, DeBlauw B, Glumac N, Dutton C, Elliott G (2010) A practical approach to piv uncertainty analysis. In: 27th AIAA aerodynamic measurement technology and ground testing conference, Chicago, Illinois. pp 4355

Lee BKH (1990) Oscillatory shock motion caused by transonic shock boundary layer interaction. AIAA J 28(5):942-944

Lumley JL (1967) The structure of inhomogeneous turbulent flows. In: Yaglam AM, Tatarsky VI (eds) Proceedings of the international colloquium on the fine scale structure of the atmosphere and its influence on radio wave propagation. Doklady Akademii Nauk SSSR, Nauka

Masini L, Timme S, Peace AJ (2018) Scale-resolving simulation of shock buffet onset physics on a civil aircraft wing. In: Applied aerodynamics conference, 24-26 July, Bristol, United Kingdom (2018)

McDevitt JB, Okuno AF (1985) Static and dynamic pressure measurements on a NACA 0012 airfoil in the AMES high Reynolds number facility. Tech Paper 2485. NASA

Ohmichi Y, Ishida T, Hashimoto A (2018) Modal decomposition analysis of three-dimensional transonic buffet phenomenon on a swept wing. AIAA J 56(10):3938-3950

Poplingher L, Raveh DE, Dowell EH (2019) Modal analysis of transonic shock buffet on 2D airfoil. AIAA J 3:1-16

Raffel M, Willert CE, Scarano F, Kähler CJ, Wereley ST, Kompenhans J (2018) Particle image velocimetry: a practical guide. Springer, Berlin

Roos F (1985) The buffeting pressure field of a high-aspect-ratio swept wing. In: 18th fluid dynamics and plasmadynamics and lasers conference, Cincinnati, OH. pp 1609

Rowley CW, Dawson STM (2017) Model reduction for flow analysis and control. Annu Rev Fluid Mech 49:387-417

Schmid PJ (2010) Dynamic mode decomposition of numerical and experimental data. J Fluid Mech 656:5-28

Taira K, Brunton SL, Dawson STM, Rowley CW, Colonius T, McKeon BJ, Schmidt OT, Gordeyev S, Theofilis V, Ukeiley LS (2017) Modal analysis of fluid flows: an overview. AIAA J 55(12):4013-4041

Taira K, Hemati MS, Brunton SL, Sun Y, Duraisamy K, Bagheri S, Dawson STM, Yeh C-A (2020) Modal analysis of fluid flows: applications and outlook. AIAA J 58(3):998-1022

Trefethen LN, Bau III D (1997) Numerical linear algebra, vol 50. SIAM, Philadelphia

Tu JH, Rowley CW, Luchtenburg DM, Brunton SL, Kutz JN (2014) On dynamic mode decomposition: theory and applications. J Comput Dyn 1(2):391-421

Westerweel J (1993) Digital particle image velocimetry: theory and application. PhD thesis, Delft University

Westerweel J (1997) Fundamentals of digital particle image velocimetry. Meas Sci Technol 8(12):1379

Westerweel J (2000) Theoretical analysis of the measurement precision in particle image velocimetry. Exp Fluids 29(1):S003-S012

Xiao Q, Tsai M, Liu F (2006) Numerical study of transonic buffet on a supercritical airfoil. AIAA J 44(3):621-628

Publisher's Note Springer Nature remains neutral with regard to jurisdictional claims in published maps and institutional affiliations. 